\title{
Four decades of European geomagnetic secular variation and acceleration
}

\author{
Giuliana Verbanac $\left({ }^{1}\right)$, Monika Korte $\left(^{2}\right)$ and Mioara Mandea $\left({ }^{2}\right)$ \\ (') Faculty of Science, University of Zagreb, Croatia \\ $\left({ }^{0}\right)$ Helmholtz-Zentrum Potsdam Deutsches GeoForschungsZentrum - GFZ, Potsdam, Germany
}

\begin{abstract}
Geomagnetic secular variation, the generally slow, continuous change in the core magnetic field, is characterized by occasional rapid variations known as geomagnetic jerks. Recent studies on magnetic data obtained by satellites with a good global coverage suggest that more rapid and smaller scale features than previously thought occur in the field change. We have taken advantage of the comparatively high density of geomagnetic observatories in Europe and have derived a regional model for the detailed study of secular variation and acceleration over the past four decades from 1960 to 2001 by means of improved and regularized spherical cap harmonic analysis. We show the improvements to our regional model over a global model. All the known jerks are seen in our model, but further times with rapid changes in secular variation exist. Moreover, times of zero acceleration in general do not occur simultaneously in all magnetic field components, although this nearly is the case in 1969.6 and 1982.2. Secular variation and acceleration show very dynamic patterns indicating rapid and complex causal processes in the Earth's fluid core.
\end{abstract}

Key words European secular variation - secular acceleration - geomagnetic jerks

\section{Introduction}

The geomagnetic field observed at the Earth's surface contains components internal (core and lithospheric fields) and external (ionospheric and magnetospheric fields) in origin. These various contributions interact and vary on large temporal and spatial scales, the lithospheric field alone, over time scales of decades to centuries. The short variations, with periods from seconds to the solar cycle, are

Mailing address: Dr. Giuliana Verbanac, Faculty of Science, University of Zagreb, Department of Geophysics, Horvatovac bb, Zagreb 10000, Croatia; e.mail: Verbanac@irb.hr mainly caused by external sources. The temporal variation of the dominant part of the Earth's magnetic field, the core field, on time scales from years to decades and longer, is referred to as secular variation (henceforth SV). One intriguing characteristic of $\mathrm{SV}$ is its rapid change in the trend occurring on short-time scales, from months to years. These events, known as geomagnetic jerks (Courtillot et al., 1978; Courtillot and LeMouël, 1984; Mandea et al., 2000 and references therein), are still not completely understood. They were first detected in time series of the magnetic east component, and due to the sparse global distribution of magnetic observatories their true spatial nature (global or regional; effects in other field components) could not be clearly determined. The occurrence of a recent magnetic jerk, captured by the new magnetic satellite data with high resolution global coverage, for the first time provided a clear picture, showing the regional nature of a 
jerk that was most obvious in the vertical component (Olsen and Mandea, 2007a). The signature of geomagnetic jerks is an abrupt change, mostly including a change of sign, in the secular acceleration (henceforth SA), the second-order magnetic field derivative.

The spatial and temporal changes of the geomagnetic field have been described by various kinds of models, on both global and regional scales. Among the global modeling techniques, the most widely considered is Spherical Harmonic Analysis (SHA). This method has been used, for example, to specify the International Geomagnetic Reference Field (e.g. Macmillan et al., 2003). Some other global models have recently been produced, based on ground and satellite data, like the Comprehensive Model CM4 (Sabaka et al., 2004), C FM (Wardinski and Holme, 2006), CHAOS (Olsen et al., 2006), or the GRIMM model (Lesur et al., 2008). After about 2000, when a good global coverage of magnetic data started to become available from the satellites Ørsted, CHAMP and SAC-C, good results can be obtained from global SH models. Before this date, higher spatial resolution can be obtained by local models when the magnetic field is described only over a given region of the Earth's surface for which high density data exist.

Such regional models of the core field and its SV are commonly derived using Spherical Cap Harmonic Analysis, SCHA, (Haines, 1985) and improvements thereof. The original SCHA method has been widely used to describe the magnetic field over different regions (Garcia et al., 1991; Torta et al., 1992; De Santis et al., 1997; Haines and Newitt, 1986; Kotzé, 2001). Although satisfactory results were obtained, a few problems were also encountered and pointed out. For example, when the cap becomes smaller, a high degree expansion is needed to describe the large wavelengths if expansion cannot be reached. Recently, two approaches to overcome some of the problems have been proposed. One is to apply a physical method of regularization (Korte and Holme, 2003), the other is the Revised SCHA, named R-SCHA (Thébault et al., 2006) which allows data from different altitudes to be taken into account. A version to apply R-SCHA to data from only one level on or above the Earth's surface has just become available at the time of writing (Thébault, 2008).

New attempts to develop regional models are currently based on wavelet analysis (Holschneider et al., 2003; Chambodut et al., 2005). This approach, however, is still in a developing phase.

In this study, we have used the regularization proposed by Korte and Holme (2003) together with an improved (Verbanac, 2007) SCH regional modeling approach to investigate in detail the SV over Europe, the region covered most densely by geomagnetic observatories, for four decades (1961-2002) where several jerks had been identified in the east component time series (Alexandrescu et al., 1995; Macmillan, 1996; Mandea et al., 2000). The data basis for this study was European observatory annual means, which we had studied in detail in a previous work (Verbanac et al., 2007a), assessing their quality and eliminating external field contributions as well as possible.

This paper is organized as follows. The data used in this study, with their distribution, are presented in Section 2. To model them we have applied the continuous, regularized SCHA. In order to better understand the model behavior with respect to the mathematical-short comings of the method, as well a the data distribution, a few parameters in the modeling process were tested first (spherical cap harmonic angle, maximal degree of the spherical cap harmonic expansion, temporal splines, norms, regularization factors) to find the appropriate ones (Section 3). The results from the preferred model in terms of SV and SA features are presented in Section 4. In Section 5 we discuss the improvements brought by our regional model when compared with a global one. We show that rapid changes in the SV and SA over Europe are well described by this new regional model.

\section{Data and processing}

The data set comprised the annual mean values of $X, Y$ and $Z$ components of the core (main) field at 46 European observatory locations, obtained from the World Data Center Edinburgh (http://www.geomag.bgs.ac.uk/gifs/an- 
nualmeans.HTML). Short-period variations of external origin exist in all annual means, and as Verbanac et al. (2007b) and the references therein conclude, means of an empirical approach as described in detail by Verbanac et al. (2007a). This approach is based on the clearly homogeneous external field variation pattern seen for the whole region.

Indeed, Verbanac et al. (2007a) have shown that a better reduction of the external field is achieved when applying this method compared to the use of the the CM4 external and induced description.

The observatory time series of $X, Y$ and $Z$ components, after subtracting the core field as predicted from the CM4 model and constant offsets to account for the lithospheric field, were median averaged in each year to obtain the external magnetic field signals typical for the studied region. This averaged external field approximation time-series was subtracted from each observatory data series. Secular variation (SV) at each location was thereafter calculated as the difference between two successive annual mean values (AM) for each component (e.g. $\left.S V_{1981.0}=A M_{1981.5}-\mathrm{AM}_{1980.5}\right)$.

The corrected data set was further examined by plotting these SV time series for each observatory and each component over the time span 1961-2001. These showed that there was a significant difference in data quality among the 46 observatories. Some of the SV series look significantly more scattered than others, sometimes even showing very sharp features. It is very difficult to find an objective criterion to discard individual values in scattered time series. However, if the scatter is normally distributed and not some systematic error it will only add to the uncertainty of the model parameters and not affect our deductions. We therefore do not discard the data from observatories with obvious scatter. However, the time series of three observatories (Pendeli - PEG, San Fernado SFS and Almeria - ALM) show very high scatter of SV in the order of $20 n T y r^{-1}$, including some extremely large and sudden changes. We discarded these data series in the modeling.

Unfortunately the three excluded observatories are located on edges of the investigated region. Moreover, a large area in the north-west- ern part is void of observatories. So, we have decided to include synthetic data for some «synthetic observatories» to minimize the edge effects and maximize the reliable region of the model. The locations of these synthetic observatories were carefully chosen: they must not be situated too close to the cap boundary, and not too close to the real observatories. Thus, an improvement in data distribution without a significant influence on the model prediction at the observatory places can be reached, as we will demonstrate in Section 4. A map indicating all used observatories, real and synthetic, is shown in fig. 1. A table linking the numbers from fig. 1 to the IAGA codes and names of the observatories is given in table I. The synthetic data series for the 11 «synthetic observatories» were obtained from the CM4 model which covers the full time interval of interest, namely the period 1961-2002. The core field components were estimated for each year by using the spherical harmonic expansion up to degree/order 13. No external field contributions were considered. Similarly as for the real observatory data, SV at each location was thereafter calculated as the difference between two successive core field values.

The final data set amounts to 4857 real observatory secular variation values, and $6210 \mathrm{da}-$ ta when including the synthetic time series from the synthetic observatories.

\section{Modeling method and parametrization}

In a source-free region, the magnetic field $\mathbf{B}$ can be represented as the negative gradient of the scalar potential $\Phi, \mathbf{B}=-\nabla \Phi$, where $\Phi$ has to satisfy the Laplace's equation: $\nabla^{2} \Phi=0$. The global solution is obtained by the well-known method of spherical harmonic analysis (see e.g. Langel (1987) for a detailed description) and is given in terms of Legendre polynomials in colatitude and trigonometric functions in longitude.

When observations are available only over a small portion of the Earth a similar method, developed by Haines (1985) and named spherical cap harmonic analysis (SCHA), can be applied for a spherical cap of half-angle $\theta_{0}$. The gener- 


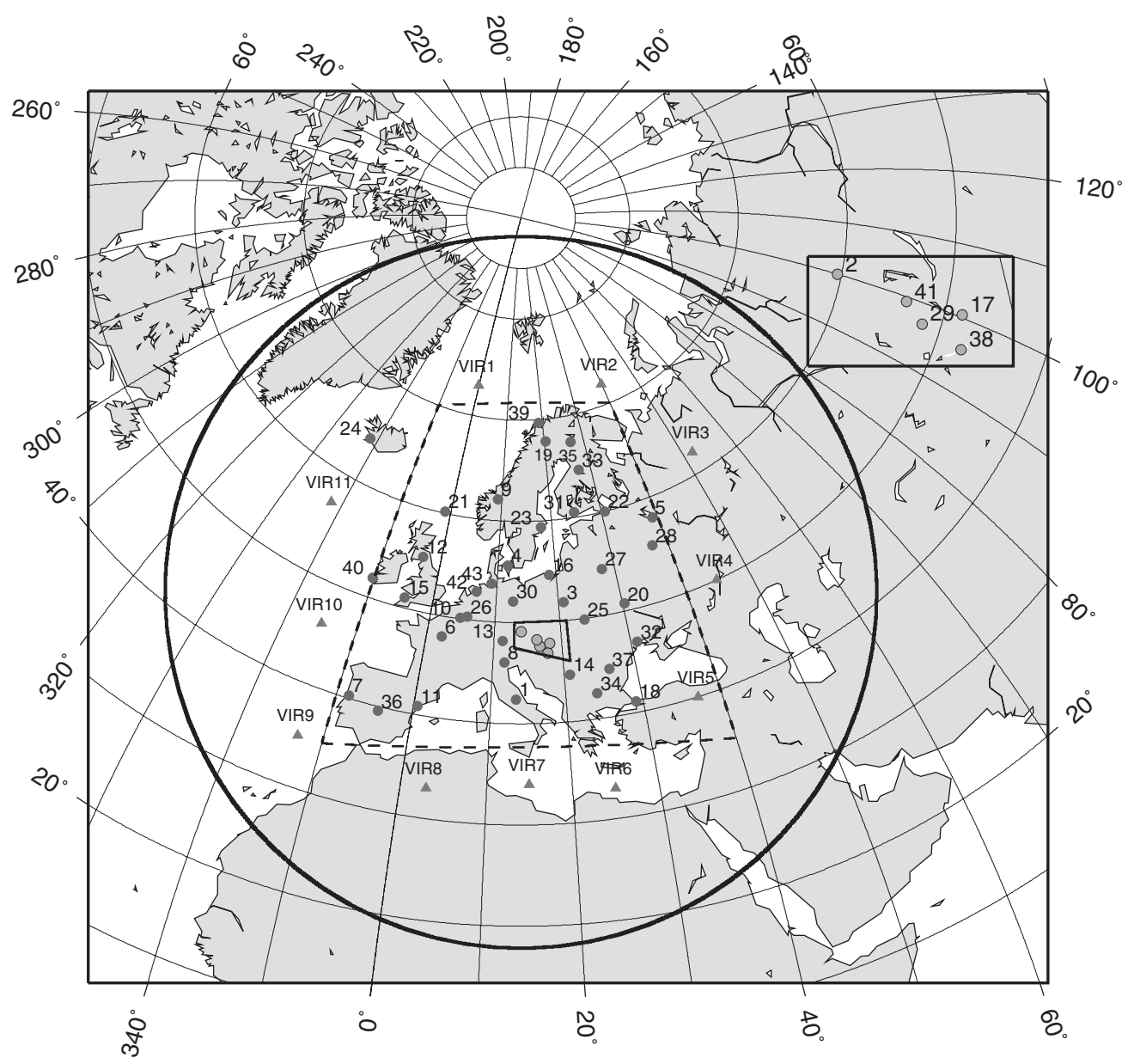

Fig. 1. Locations of the geomagnetic observatories (full circles) and synthetic observatories (triangles) for which synthetic data sets are computed from CM4 model. The geomagnetic observatories are labeled by a number corresponding to the alphabetical order of their IAGA code (see table I). The large circle shows the border of the spherical cap of $\theta_{0}=35^{\circ}$ used for the SCHA-modeling. The dashed boundary indicates the region of interest, over which all maps and animations are produced. The green circles and inset are used to label four close observatories. Equidistant azimuthal projection.

al solution of Laplace's equation in the case of SCHA for one epoch is:

$$
\begin{aligned}
& \Phi(r, \theta, \phi)=R_{E} \sum_{k=0}^{k_{\max }} \sum_{m=0}^{k}\left(\frac{R_{E}}{r}\right)^{n_{k}+1} \\
& \left.\left[g_{k}^{m} \cos (m \phi)+h_{k}^{m} \sin (m \phi)\right] P_{n_{k}}^{m}(\cos \theta)\right]
\end{aligned}
$$

The potential, a function of radius $r$, co-latitude $\theta$ and longitude $\phi$, is developed into a se- ries of associated Legendre functions $\left\{P_{n_{k}}^{m}\right\}$ with non-integer degrees $n_{k}$ and integer orders $m$. The integer index $k$ is used to order the functions and the SCHA coefficients, $\left\{g_{k}^{m}, h_{k}^{m}\right\}$.

The values of $n$ have to be non-integer in order to satisfy certain boundary conditions for the spherical cap instead of a whole sphere. The order of the Legendre functions remains integer, ensuring the continuity of the potential in 
Table I. Geomagnetic observatories considered in the present study.

\begin{tabular}{|c|c|c|c|c|}
\hline $\mathrm{Nr}$ & IAGA code & Station & Geographic Long $\left(^{\circ}\right)$ & Coordinates Lat $\left({ }^{\circ}\right)$ \\
\hline 1 & $\mathrm{AQU}$ & L'Aquila & 13.32 & 42.38 \\
\hline 2 & $\mathrm{BDV}$ & Budkov & 14.02 & 49.07 \\
\hline 3 & BEL & Belsk & 20.80 & 51.83 \\
\hline 4 & BFE & Brorfelde & 11.67 & 55.63 \\
\hline 5 & $\mathrm{BOX}$ & Borok & 38.97 & 58.03 \\
\hline 6 & CLF & Chambon-la-Foret & 2.27 & 48.02 \\
\hline 7 & $\mathrm{COI}$ & Coimbra & 351.58 & 40.22 \\
\hline 8 & CTS & Castello Tesino & 11.65 & 46.05 \\
\hline 9 & DOB & Dombas & 9.12 & 62.07 \\
\hline 10 & DOU & Dourbes & 4.60 & 50.10 \\
\hline 11 & EBR & Ebro & 0.50 & 40.82 \\
\hline 12 & ESK & Eskdalemuir & 356.80 & 55.32 \\
\hline 13 & FUR & Fuerstenfeldbruck & 11.28 & 48.17 \\
\hline 14 & GCK & Grocka & 20.77 & 44.63 \\
\hline 15 & HAD & Hartland & 355.52 & 51.0 \\
\hline 16 & HLP & Hel & 18.82 & 54.60 \\
\hline 17 & HRB & Hurbanovo & 18.18 & 47.87 \\
\hline 18 & ISK & Istanbul - Kandilli & 29.07 & 41.07 \\
\hline 19 & KIR & Kiruna & 20.40 & 67.80 \\
\hline 20 & KIV & Kiev - Dymer & 30.30 & 50.72 \\
\hline 21 & LER & Lerwick & 358.82 & 60.13 \\
\hline 22 & LNN & Leningrad - Voiekovo & 30.70 & 59.95 \\
\hline 23 & LOV & Lovo & 17.83 & 59.35 \\
\hline 24 & LRV & Leirvogur & 338.30 & 64.16 \\
\hline 25 & LVV & Lvov & 23.75 & 49.90 \\
\hline 26 & MAB & Manhay & 5.68 & 50.30 \\
\hline 27 & MNK & Minsk - Pleshchenitzi & 27.88 & 54.50 \\
\hline 28 & MOS & Krasnaya - Pakhra & 37.32 & 55.47 \\
\hline 29 & NCK & Nagycenk & 16.72 & 47.63 \\
\hline 30 & NGK & Niemegk & 12.68 & 52.07 \\
\hline 31 & NUR & Nurmuijarvi & 24.65 & 60.52 \\
\hline 32 & ODE & Odessa - Stepanovka & 30.88 & 46.78 \\
\hline 33 & OUL & Oulujarvi & 27.23 & 64.52 \\
\hline 34 & PAG & Panagjuriste & 24.18 & 42.52 \\
\hline 35 & SOD & Sodankyla & 26.63 & 67.37 \\
\hline 36 & SPT & San Pablo & 355.65 & 39.55 \\
\hline 37 & SUA & Surlari & 26.25 & 44.68 \\
\hline 38 & THY & Tihany & 17.90 & 46.90 \\
\hline 39 & TRO & Tromso & 18.93 & 69.67 \\
\hline 40 & VAL & Valentia & 349.75 & 51.93 \\
\hline 41 & WIK & Wien - Cobenzl & 16.32 & 48.27 \\
\hline 42 & WIT & Witteveen & 6.67 & 52.82 \\
\hline 43 & WNG & Wingst & 9.07 & 53.75 \\
\hline
\end{tabular}


longitude. $R_{E}$ is the mean radius of the Earth. When deriving the mathematical model by means of the linear inverse method, the classical approach is to minimize the difference between the observed data and prediction. In order to find the model with the minimum structure required by the data where any data errors are not fit too closely, a regularization can be applied.

The maximum likelihood solution is then:

$$
\hat{m}=\left(A^{T} C_{e}^{-1} A+\lambda \wedge\right)^{-1} A^{T} C_{e}^{-1 y}
$$

where $y$ is the data vector, $\mathbf{A}$ is the operator mapping model vector on to the data vector $\mathbf{m}$, $\mathbf{C}_{e}$ is the data error covariance matrix, $\wedge$ is a damping matrix and $\lambda$ is a Lagrange multiplier. We did not apply any weighting to the data, so $\mathbf{C}_{e}$ simply is the unit matrix in this case. The damping matrix is determined by the norm and the regularization is performed by minimization of certain properties of the field over the cap surface. Following Korte and Holme (2003), we used a combination of the mean square values of $\mathbf{B}^{2}$ and $(d \mathbf{B} / d r)^{2}$ over the cap as regularization norms. To provide a temporally continuous description of the field, each of the SCHA coefficients is expanded in time as a linear combination of cubic $B$-splines $B_{j}(t)$ with equidistant division of knot points:

$$
g_{n k}^{m}(t)=\sum_{j=1}^{L} \alpha_{j k m} B_{j}(t)
$$

A detailed discussion of splines and the $B$ spline basis is given by de Boor (1978).

The $a_{j k m}$ are temporal coefficients and in this case a temporal regularization is added in eq. 3.2. Korte and Holme (2003) implemented regularized temporal continuity by splines in the SCHA formalism. Following this approach, we minimize the functional:

$$
\begin{aligned}
(y-A m)^{T} C_{e}^{-1}(y-A m) & +\int\left[\lambda \int\left(B^{2}+0.1 R_{E}^{2}\left(\frac{d B}{d r}\right)^{2}\right) d \Omega\right. \\
& \left.+\tau \int\left(\left(\frac{\partial^{2} B}{\partial t^{2}}\right)^{2}+0.1 R_{E}^{2}\left(\frac{\partial^{2}}{\partial t^{2}}\left(\frac{d B}{d r}\right)^{2}\right)\right) d \Omega\right] d t
\end{aligned}
$$

Here, $\lambda$ and $\tau$ are the spatial and temporal damping constants, respectively. We multiplied $(d \mathbf{B} / d r)^{2}$ with factors 0.1 and $R_{E}$ to ensure that the two damping norms have similar contribution and units. Note that the accuracy by which the Legendre functions are computed greatly influences the SCHA results. The original subroutine for computing these functions (Haines, 1985) often encountered a lack of convergence of the series leading to incorrect values of the $\left\{P_{n k}^{m}(\cos \theta)\right\}$.

The problem arises especially for large value of degree $n_{k}$ or high values of latitude. Note that this is no intrinsic problem of SCHA method. To compute the associated Legendre function values appropriately, the algorithm proposed by Olwer and Smith (1983), which uses extended-range arithmetic subroutines, was implemented and successfully applied to model the main geomagnetic field over Europe within the time span 1960-2001 (Verbanac, 2007).

The theoretical background presented above is valid for the regional magnetic field modeling. This approach can also be applied to directly describe the secular variation. Indeed, instead of computing magnetic field coefficients based on magnetic field data, the secular variation coefficients are obtained directly from secular variation values. This has two advantages for our study. first, by considering differences of field values the constant lithospheric field contribution is eliminated. Second, by applying the temporal regularization to the second time derivative we also obtain a smooth description of SA where fast changes will show only when truly required by the data.

We investigated different SCHA parameters such as spherical cap half-angle, maximum spherical cap harmonics order and damping factors. The region to be studied has to be defined by a spherical cap well covering that area, with margins large enough to prevent, as far as possible, the influence of the cap boundary. A cap centered at $53^{\circ} \mathrm{N}$ latitude and $14^{\circ} \mathrm{E}$ longitude with the spherical cap half angle of $35^{\circ}$ defines well the region of interest, as seen in fig. 1.

The maximum spherical cap harmonics index was chosen as $k_{\max }=8$, considering that the model structure should be controlled by a tradeoff between good fit to the data and smoothing regularization constraint and not by the spheri- 


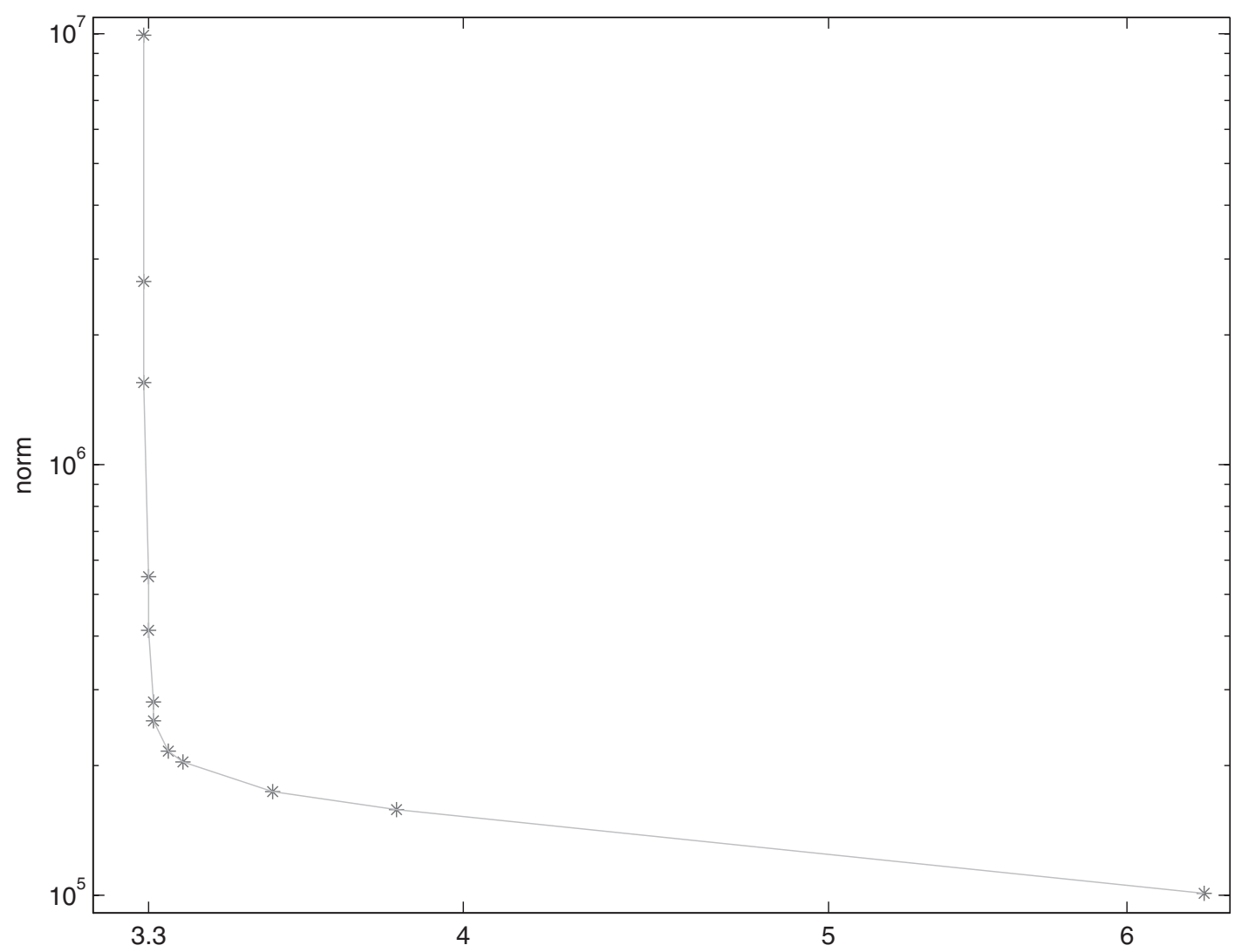

Fig. 2. Trade-off curve of the spatial norm $\left(n T y r^{-1} / 4 \pi\right)$ vs. misfit $\left(n T y r^{-1}\right)$ for EU_MIX model. Logaritmic scale.

cal expansion truncation level. For temporal modeling with splines, we chose 20 splines, that means a total of 1620 coefficients had to be calculated, 81 for each knot point.

Special attention was paid to find the best spatial $(\lambda)$ and temporal $(\tau)$ damping factors. We first investigated a broad interval of spatial and temporal damping factors. Over a wide range the misfit changes very little with temporal damping, and we kept the largest temporal factor before the misfit starts to change more significantly for a broad range of spatial factors. Then, keeping temporal damping factor constant we analyzed the misfits for a wide range of spatial damping factors and looked at the plot of the norm value against misfit, a trade-off curve. The optimal spatial damping factor was taken as the «knee» of this trade-off curve (see fig. 2).

The values used for our preferred models presented in the following are $\lambda=5 \times 10^{-3} n T^{-2} y r^{2}$, $\tau=5 \times 10^{-1} n T^{-2} y r^{6}$. With our data set and chosen parameters there was no need to apply any additional numerical damping.

The models computed and presented in the following simultaneously describe the secular variation and secular acceleration. Before discussing specific features of these two core field characteristics, in the region of interest we compare in detail three models: the regional models with and without data from the synthetic observatories and the global CM4 model (Sabaka et 
a)

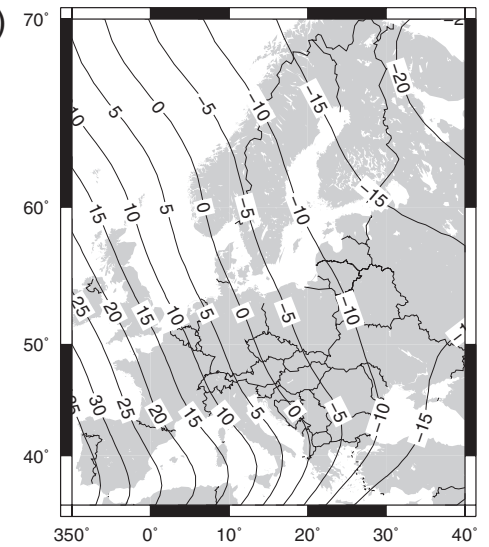

b)

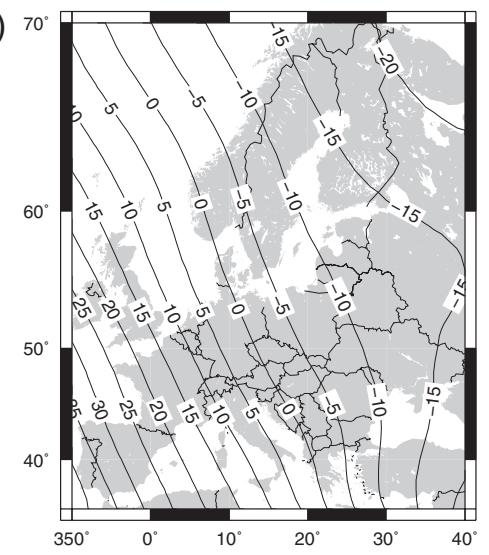

c)

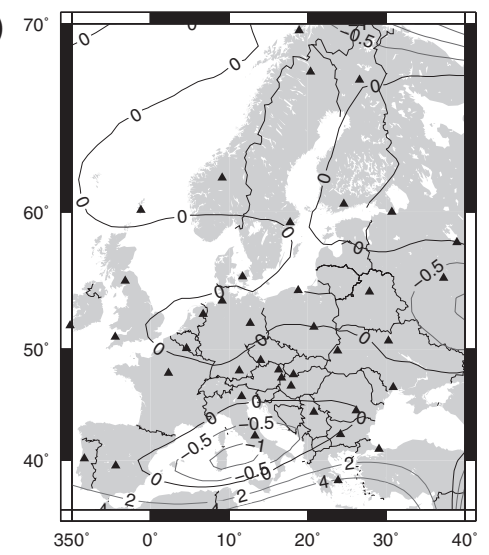

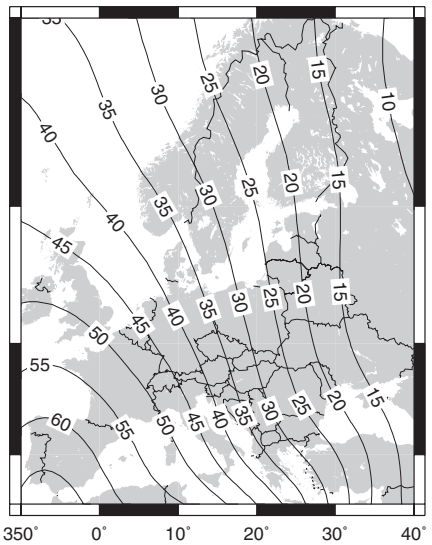
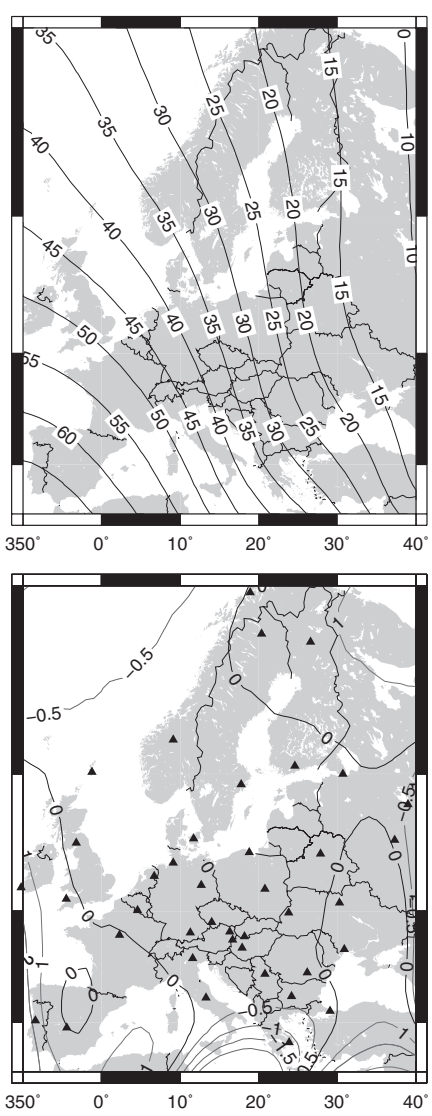
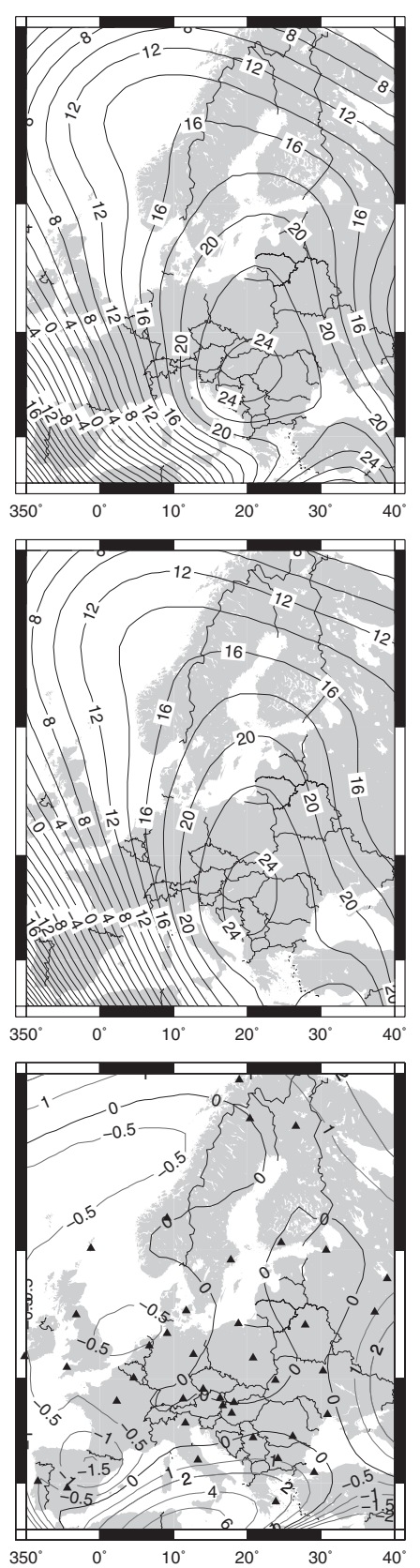

Fig. 3a-c. Maps of the $X$ (left), $Y$ (center) and $Z$ (right) secular variation components at epoch 1980.5 obtained from: (top) SCHA at 43 observatory locations (model EU_OBS) (middle) SCHA at 43 observatory locations complemented with eleven synthetic observatories (model EU_MIX). Maps of differences between EU_MIX and EU_OBS, (EU_MIX-EU_OBS), model values are also shown (bottom). Triangles show the observatory locations. Units $n T y r^{-1}$. Mercator map projection. 
al., 2004). The following discussion of field characteristics is based on our preferred regional model based on the results of this comparison.

\section{Results}

\subsection{Models}

Figure 3 presents contour maps of two computed secular variation models, one based on data from 43 European observatories (EU OBS), and one (EU-MIX) based on the same data set complemented with synthetic data computed at 11 additional synthetic sites, from the CM4 global model. The model values for all maps were calculated on a regular grid of $1^{\circ} \times 1^{\circ}$ in the region of interest, indicated by a dashed line in fig. 1. We show maps for the epoch 1980.5, as representative example to illustrate the following discussion of differences between the models and fit to the data. It should be kept in mind that the model region is larger than the region of interest. Artificial effects at the edges of the considered region are not very prominent, even without synthetic observatories. The isolines are slightly smoother in the case of EU_MIX.

For a better insight on the influences of the synthetic observatories, we calculated the differences between the two models, EU_OBS and EU_MIX, and the results are presented in the bottom panels of fig. 3. The strongest differences appear near the edges, predominantly at the southern border. This could be explained by a lack of observatory data in the south-west and south-east parts of the region, where the SV gradients are relatively strong. Very small differences are found in the north-west part despite the poor data coverage. The SV gradients are smaller there. Indeed, this is the only area where a real observatory exists outside our region of interest (LRV on Iceland), but within the modeling region, so that these data were used in the modeling. Over the whole central part of the studied area the differences take values of less than $0.5 n T y^{r-1}$. We can conclude that the synthetic data from synthetic observatories minimize the numerical instability, without in- fluencing the model performance in the region covered by real data (where we strive for an improved regional model compared to the global one). Comparing our regional model EU_MIX to $\mathrm{CM} 4$, the contour maps look quite similar (top panels of figs. 3 and 4, respectively). For a more detailed investigation of SV structures, the differences between the CM4 and EU_MIX model were calculated (bottom panels of fig. 4). As expected, the differences are somewhat larger, on average with values in the order of 1 to 2 $n T y r^{-1}$, but reaching values of up to $7 n T y r^{-1}$ in certain years, regions and components. Some details about these differences will be discussed in the following.

\subsection{Model quality}

Performance and reliability of different models can be judged by the fit to the data, overall and for individual observatories and components. The rms misfit of the reference model CM4 to all the real observatory data is $4.0 n T y r^{-1}$. Both our regional models achieve a somewhat better fit. It is worth noting that the overall $\mathrm{rms}$ misfit is reduced strongest in EU_ MIX, the model stabilized at the borders by synthetic data. The misfit amounts to $3.6 n$ nyr $^{-1}$ for EU_OBS, and is $3.3 n T y r^{-1}$ in EU_MIX. We therefore consider EU_MIX as our best regional model and will not consider EU_OBS any further in the following. The rms misfits of CM4 and EU_MIX for the individual observatories are displayed in Figure 5. With few exceptions the rms misfits are somewhat larger in the case of the global model in all three components.

In the $X$ component, the rms misfits at many locations are less than $2 n T y r^{-1}$, some are between 2 and $4 n T y r^{-1}$ and only a few are larger then $4 n T y r^{-1}$. The worst fits (rms misfit higher than $7 n T y r^{-1}$ ) are found at MOS and THY, and the best at NGK. The largest differences between the global and the regional model amount to $\sim 1.4 n T y r^{-1}$ at TRO and COI.

For the $Y$ component, there are a number of observatories with very low rms misfits, around $1 n T y r^{-1}$. The best fit is again found for NGK. Only at some locations are the rms misfits larg- 
a)

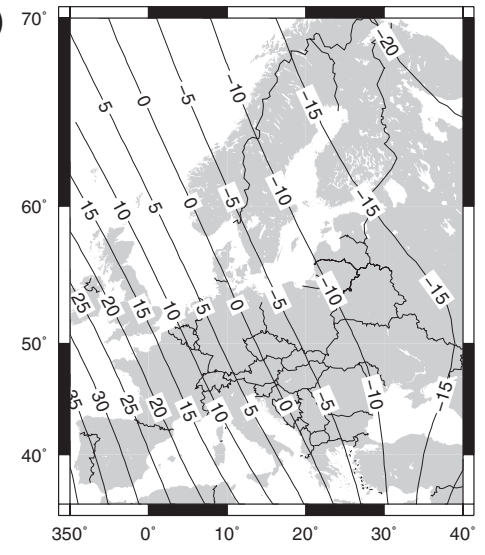

b)

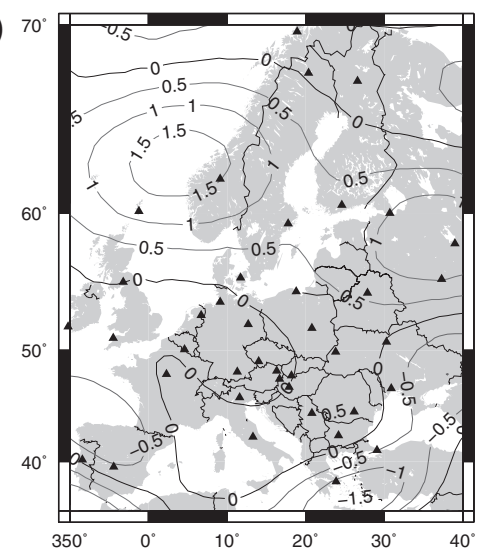

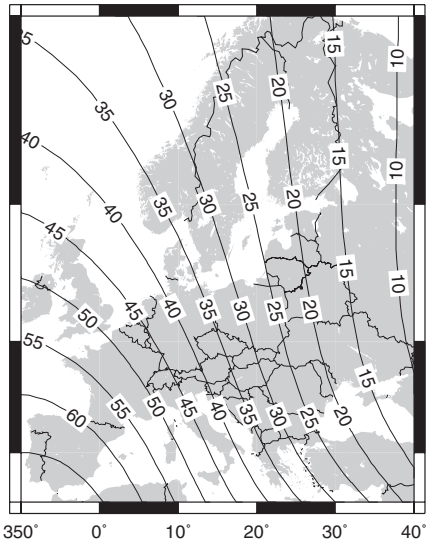
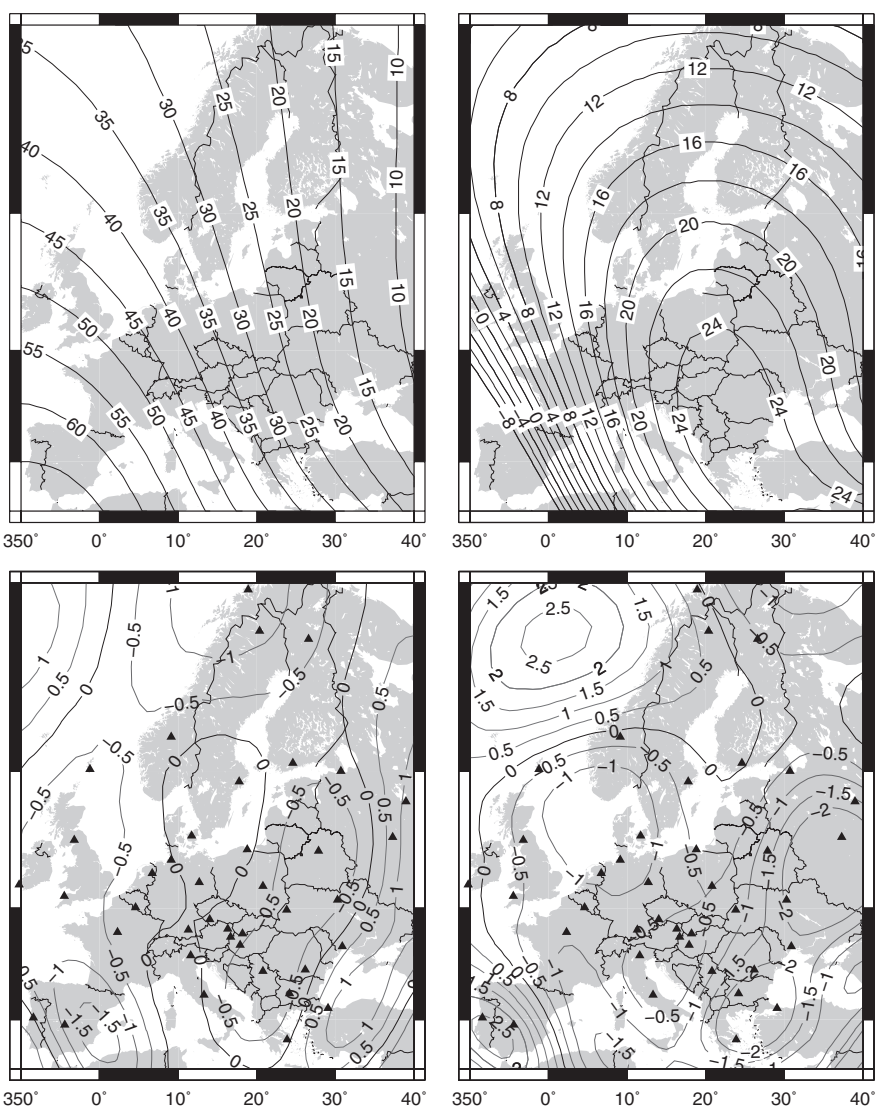

Fig. 4a-b. Maps of the $X$ (left), $Y$ (center) and $Z$ (right) secular variation components at epoch 1980.5 obtained from the CM4 model (top). Maps of differences between EU_MIX and CM4 models, (EU_MIX- CM4), are also shown (bottom). Units $n T y r^{-1}$. Mercator map projection.

er than $2 n T y r^{-1}$. Here, two observatories are outstanding, namely HAD and MNK, with rms misfits larger then $8 n T y r^{-1}$. At MNK also the difference between the two models is largest (more than 1 nTyr $r^{-1}$ ).

Slightly larger rms misfits are more common in the $Z$ component. There are several observatories with rms larger then $6 n T y r^{-1}$ (COI, ISK, KIR, LVV, MAB, MNK, TRO), while the lowest rms is again noticed at NGK. The most distinct discrepancy between global and regional model rms misfit is found at COI and amounts to almost $2 n y r^{-1}$.

The bar chart showing the overall rms mis- fit per observatory in the bottom panel of fig. 5 reveals rms misfits mostly below $2 n y r^{-1}$, but also many values larger then $4 n T y r^{-1}$. The largest difference between two models is found at COI, amounting to $\sim 1.5 n T y r^{-1}$.

The rms differences between the two models are largest at those locations where the misfit is largest. Indeed, the regional model does fit those data better, but the large misfit might also indicate high uncertainties or problematic data at such observatories. At the synthetic observatories (not shown on the charts of fig. 4), the rms misfits of EU_MIX in all components are similar. With values below or 
a)

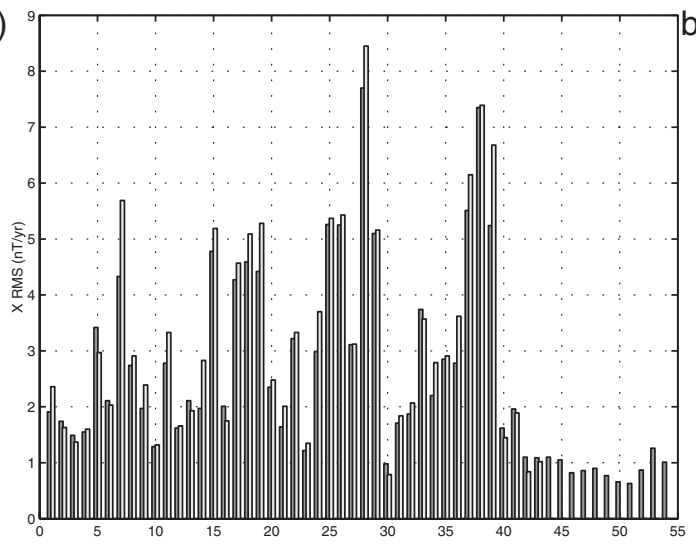

c)

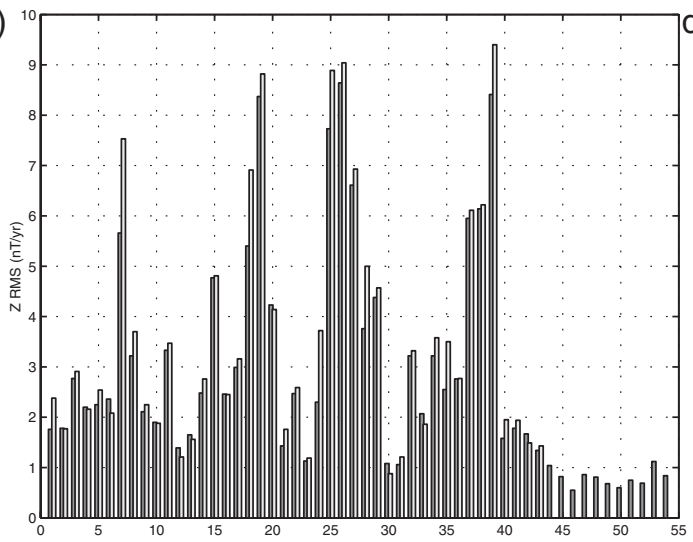

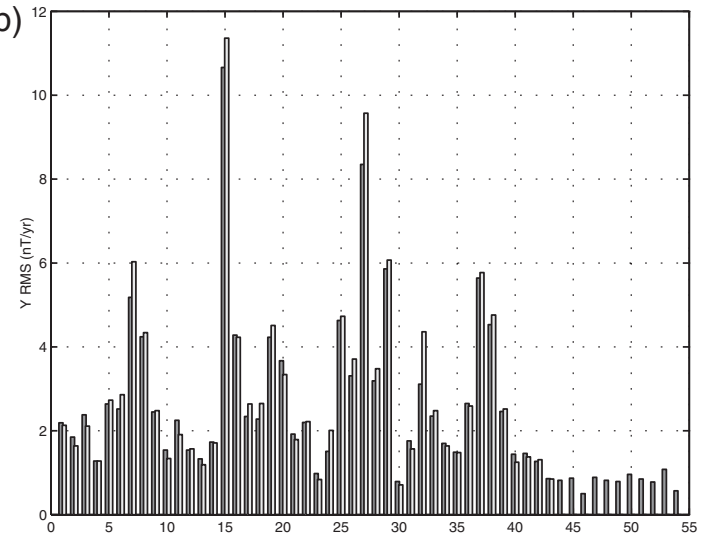

d)

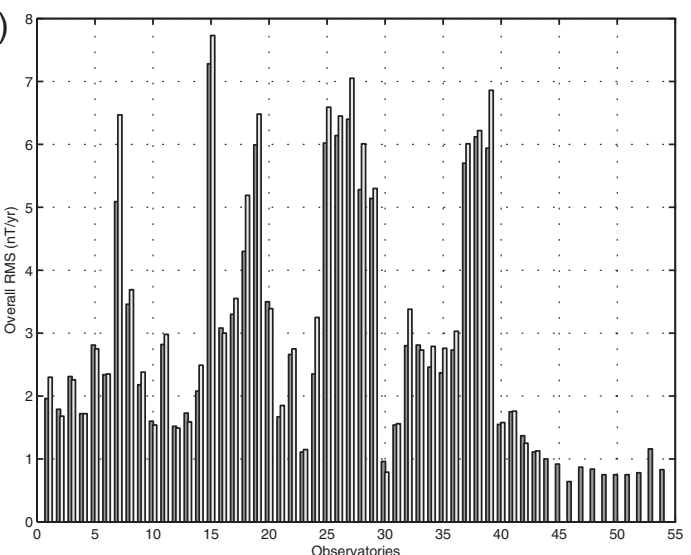

Fig. 5a-d. The $X, Y, Z$ and the overall rms misfit at each observatory locations from the regional, EU_MIX model (black) and the global, CM4 model (white).

around $1 n T y r^{-1}$ this demonstrates the close agreement of the regional model with the global model in this peripheral area where no real data are available. We have to examine some individual time series in detail in order to be sure that our regional model is not giving a better fit only because it is fitting unreliable data more closely. The results at three locations, NGK, TRO and COI, are presented in fig. 6 as representative examples for different situations. The data series are shown together with the continuous CM4 and EU_MIX predictions for that location. Figure 6 a shows an example for observatory data with high quality (NGK), which fit very well by both the regional and the global model over the whole time span and in all three components. Figure $6 \mathrm{~b}$ shows the time series at TRO, where large rms misfits and significant differences between the models are found in $X$ and $Z$. Some scatter in the data is obvious, which is not fit by any of the models. Both models fit the general trends well, but EU_MIX fits some of the patterns on intermediate time-scales more closely than CM4. For instance, the outstanding structure observed between 1971 and 1973 in $X$ is better described by the regional model. That feature is quite sharp to be a natural effect, but similar behav- 
a)
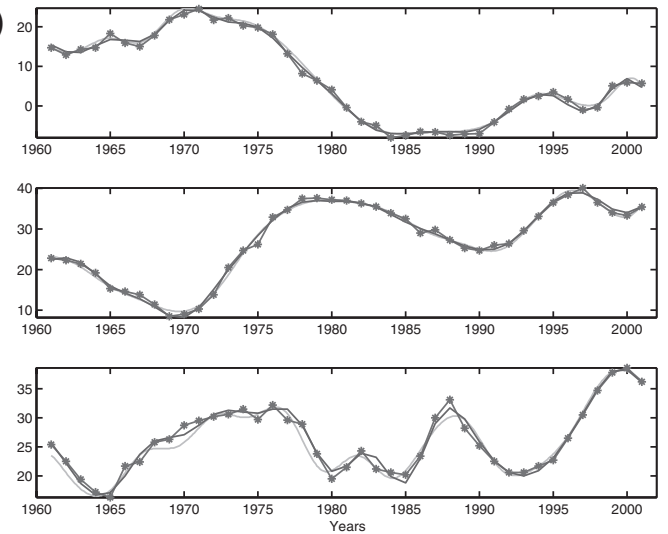

c)
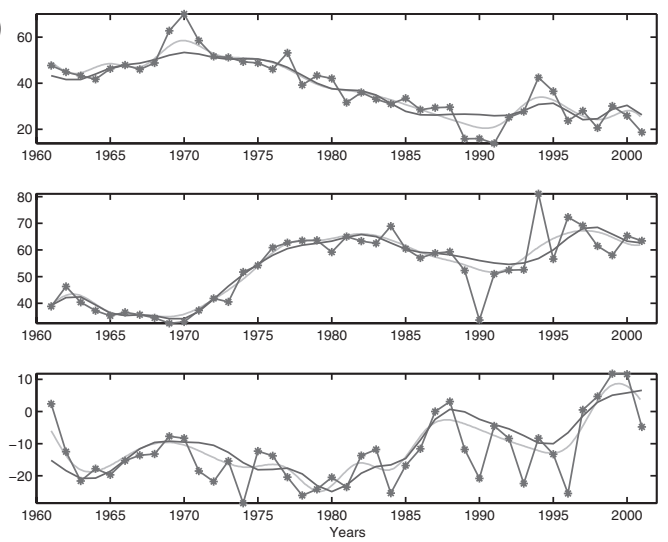

ior is noticed also in the time series at the neighboring KIR observatory, and some other observatories on the western side of the region of interest (see for example fig. 6c). An error in a particular annual mean gives successive SV points which are above and below the trend, respectively, or vice versa. The reason of the outstanding structure at $1971-1973$ in $Z$ may be possibly due to this effect, but does not influence our model in any significant way.

Figure $6 c$ is an example of large differences in both $X$ and $Z$ between the EU_MIX and CM4 models (COI observatory). Again, at some times the data are quite scattered and it is difficult to judge with absolute certainty whether the regional model is fitting the actual secular variation better. However, from examining the fit to all the data series we sup- b)
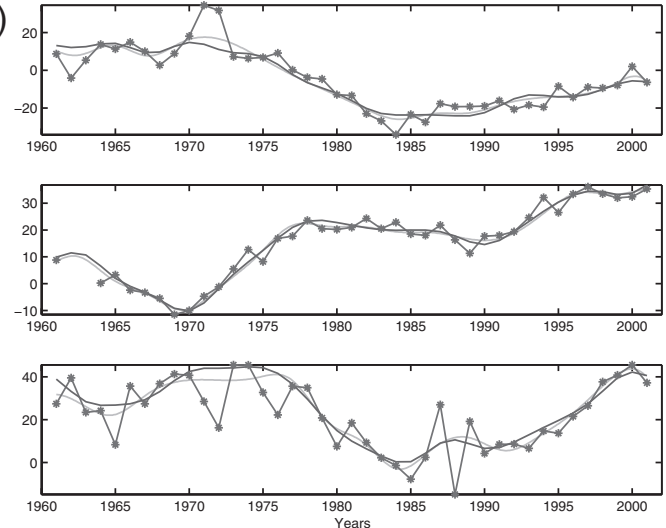

Fig. 6a-c. Time-series for $X$ (first row), $Y$ (second row) and $Z$ (third row) SV components in $n T y r^{-1}$, at (a) NGK, (b) TRO and (c) COI locations (see fig. 1 for the locations). The time series are corrected observatory data (thick black), computed values from the EU_MIX model (gray) and CM4 model (black), respectively. pose that this is the case. Some patterns in the scattered data, e.g. those resembling jumps in $Y$ in 1990 and 1994, are observed similarly (with different magnitude) at some other observatories (see fig. 6b). They appear too sharp to be secular variation features, but very likely contain contributions from external fields, that still have not been eliminated despite our efforts.

In summary, there is no indication that our model is fitting any unreliable data too closely. The examination of the time series also shows the capability of EU_MIX to model the data well even at the beginning and the end of the considered time span, where edge effects of the spline representation can generally introduce larger uncertainties in continuous models. 


\subsection{European secular variation and secular acceleration}

We use the EU_MIX model to study in detail the SV and SA over Europe. Animations of both SV and SA in $0.1 y r$ time steps for the three orthogonal magnetic components are available as supplemental material. This time step interval has been chosen mainly to obtain a reasonable speed for the animation. Although the temporal resolution of the data is one year, our continuous model provides a smooth interpolation between the data epochs. Figure 7 shows the SA around epoch 1970 as an example where large areas of zero acceleration for each component are detected. The following discussions, however, are based on the animations. All components show complex patterns, with some varying extrema. We only discuss the large-scale variations of SA, because smallscale features of the model, particularly in $X$ and $Z$, might be slightly contaminated by external field influences not completely removed by our approach. The $\mathrm{X}$ component shows a general ENE-WSW trend in SV changes. The SV isoline motion is rather constant for the Western European area, even though the direction changes from time to time. These changes are clear for the center Europe, around 1969, 1983, 1994 and 1996, and much more accentuated in the North-Eastern part. For the SA, after a rather nearby zero acceleration in 1965, the first epoch with significantly large values (some $\left.4 n T y r^{-2}\right)$ in South-Eastern and Northern Europe, is beginning of 1969. However, they are rapidly decreasing, and at the beginning of the next year, nearby zero acceleration values cover large part of Europe. The next deceleration regime reaches some extreme values around 1976 (some $-5 n T y r^{-2}$ ), and exists until 1980, again a characteristic mainly for South-Eastern Europe. During the next years the SA values are rather small, with a largely vanishing SA at the beginning of 1985, but with an accelerating tendency, with a maximum around 1992, this time in the South-Western part. After a rapid decrease, the SA accelerates again, with notably large values all over Europe around 1997. To summarize this component behavior, it appears that for SV and the SA the general trend in changes is ENE-WSW, with dynamic SA foci mainly in the South-Eastern and South-Western Europe, and clear periods over which the changes in magnetic field accelerate or decelerate. These intervals are interrupted by periods over which the SA changes the sign, more or less rapidly.

The $Y$ component $\mathrm{SV}$, similar to the $X$ component, shows larger values in the Western than in the Eastern part, and this for the entire time interval. The general trend for the isoline motion is again mainly ENE-WSW. An overall increase of SV over the 41 years is observed, most strongly over the western part. Alternating east-west movements of isolines are noticed, with rather abrupt changes in the years 1961, 1969, 1978-1980, 1990 and 1995. Most of these changes reflect the geomagnetic jerks known from the $Y$ component of the European observatory data. The general large deceleration values for SA are slowly decreasing, vanishing over near all Europe towards 1969. This is one of the quietest periods, when null-curve SA remains over the whole continent for a few months. A change in SA sign appears after this, simultaneously in the South-Western and North-Eastern parts, the acceleration continuously increasing until the end of 1971, to some $7 n T y r^{-2}$. High acceleration values characterize the next years, until the middle of 1977, when again a near null acceleration is observed over the whole of Europe. Interestingly, over the next few years the SA keeps very low values, with a clear deceleration starting with the end of 1982. The deceleration remains at the some level, of a couple of $n T y r^{-2}$ until the beginning of 1989, when again a nearly zero SA characterizes the whole area. The change in sign appears rapidly, from the east. Over the next few years no clear epoch of null curves coverage can be distinguished, even if the null isoline exists, with rapid changes in its position. At the end of 1992 large acceleration values cover all area, with maximum of some $7 n T y r^{-2}$. This maximum value, as does the one observed at the end of 1971, occurs at an edge part of the investigated area. We can consider it an upper boundary level of SA in the European region. At the beginning of 1997 two clear SA foci co-exist, a positive one centered largely over Austria, and a negative one over 
a)

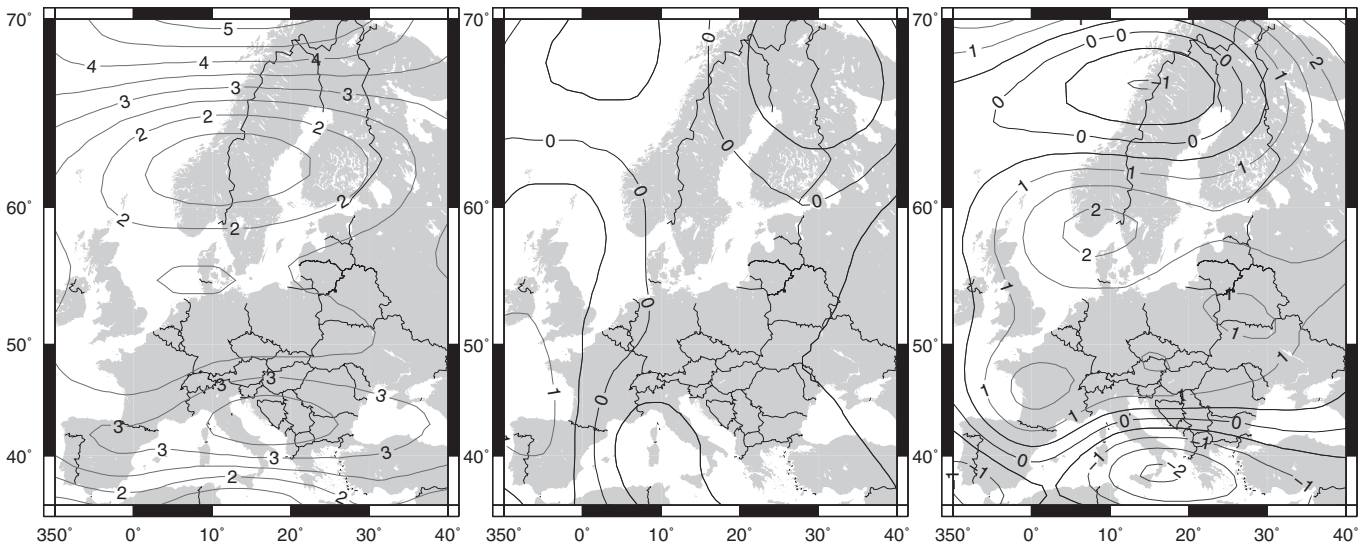

b)

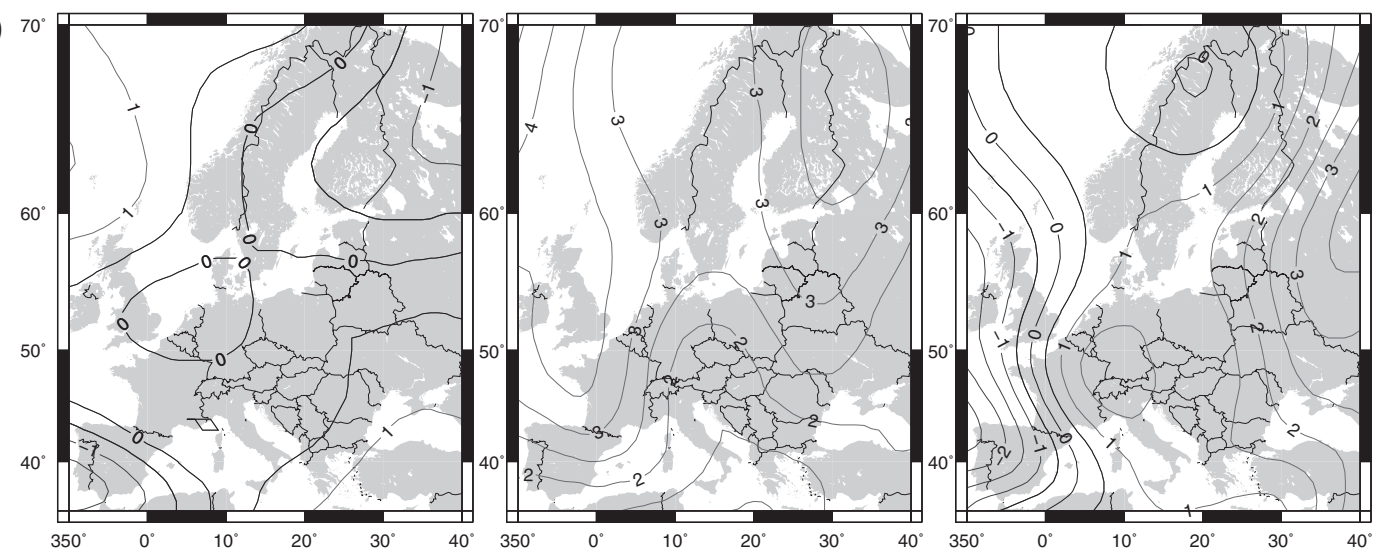

c)

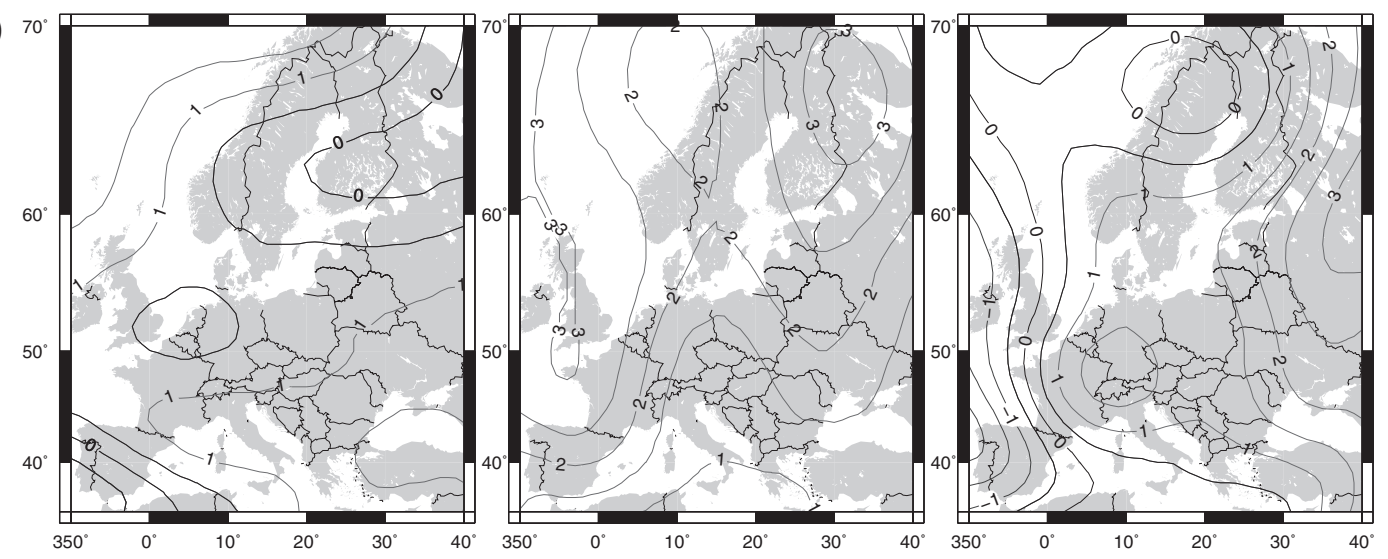

Fig. 7a-c. Maps of the $X$ (left), $Y$ (center) and $Z$ (right) secular acceleration components at epochs around 1970 obtained from EU_MIX model. The largest areas of zero SA are seen in 1969.3 for $Y$ (top), 1970.3 for X (middle) and 1970.1 for $Z$ component (bottom). Note that data resolution is one year and the decimal years reflect the smoothness quality of the model. Units $n T y r^{-2}$. Mercator map projection. 
the Ukraine. Again, at the beginning of 1999 Europe is characterized by SA values close to zero, although not everywhere. In summary it can be said for this component that the spatial patterns shown by SA around the different jerks (changes from acceleration to deceleration or vice verse) are quite different, perhaps except for the ones occurring in 1990 and 1995.

The $Z$ component SV changes are more complex than in the relatively simple ENEWSW trends seen in the $X$ and $Y$ components. Moreover, the SV behavior is more dynamic, in both time and space. It is more difficult to identify epochs when dramatic changes in the SV for $Z$ component occur over the whole region of interest. These changes are better described by the SA. The first years covered by our model indicate that the SA changes from a decelerating regime to an accelerating one, around 1963. The next important change arrives in mid-1969, when the entire area is covered by near-zero values. During the next years, SA shows a coexistence of both regimes, with a deceleration dominating all area starting with mid-1976, with some values as large as $-9 n T y r^{-2}$, until the beginning of 1979 . With this date and for the next few years again both regimes exist, the zero curve positions changing rapidly from the south-west to far north, and back. The acceleration behavior is thereafter characterized by large extreme values up to some $8 n T y r^{-2}$ around 1986 over the Northern Scandinavian region. The last decade covered by our model reveals mostly an acceleration regime nearly all over Europe. However, for the last three years a large deceleration characterizes the whole area. Although the complicated pattern of co-existing acceleration and deceleration regions over $\mathrm{Eu}-$ rope seems to show somewhat regularly alternating changes during some epochs in this component, this does not occur periodically in time.

\section{Conclusions}

We have modeled the geomagnetic field changes over Europe for the past four decades by applying a regularized SCHA approach to the SV data series from 43 geomagnetic observatories. The modeling technique also provides a smooth and detailed description of SA. Edge effects due to the data distribution have been reduced by adding 11 additional 'synthetic observatories', with synthetic data obtained from a global model. We have shown that in this way we can minimize the numerical instability, without affecting the model quality in the region covered by real data. A comparison between our preferred regional model, EU_MIX, and the global CM4 model shows generally similar behavior. However, the overall rms misfit calculated from EU_MIX is smaller than the rms obtained from $\mathrm{CM} 4$, suggesting that the new regional model gives an improved fit to the data in both time and space. Moreover, with few exceptions, the misfit for the individual observatories is smaller when the EU_MIX model is considered. Both models provide a good fit for all three components at all sites, however, over the center region of interest the differences between the EU_MIX and CM4 predictions reach maximum values of about $6 n$ Tyr $^{-1}$ in $X, 7 n T y r^{-1}$ in $Y$, and $8 n T y r^{-1}$ in $Z$.

Animations for the SV and SA allow yeld a detailed insight into their behavior on a regional scale. The SV of both X and Y components show a general ENE-WSW tendency in their general changes in direction in which the plotted contour lines are moving. For the $Z$ component, the SV changes are more dynamic, in both time and space. Exact times and spatial distribution of significant changes in SV are obtained by studying extrema and zero acceleration periods in the SA animations. Some striking features have been discussed, with mainly rapid changes for some epochs and no or very small changes for some others. We note that mainly for two epochs, 1969.6 and 1982.2, SA nearly vanishes for the whole region and in all components. A zero level in acceleration is an indication for a change of trend in the secular variation, i.e. a geomagnetic jerk. Interestingly, the EU_MIX model demonstrates its ability to show the existence of the well-known geomagnetic jerk of 1969 in all components (fig. 7), even though the null SA curves do not cover the very full region for that epoch.

It is worth noting that in general the nullSA-curves do not occur uniformly in the whole field vector over Europe. Indeed, each compo- 
nent shows some other epochs when the general SA behavior is vanishing. These epochs can be summarized as following: for the $X$ component around 1965, 1970, 1985 and 1994; for the $Y$ component rather during 1969, end 1977, 1989 and 1996; and for the $Z$ component in 1969, 1982 and 1987. Some years of these abrupt changes match the well-known epochs of geomagnetic jerks already noted in the $Y$ component of the European observatories.

The results obtained from the EU_MIX model concerning the $Y$ component are trustworthy. Those for the $X$ and $Z$ components are less robust as the data describing these field variations might still contain external and induced field influences, even after applying the approach proposed by Verbanac et al. (2007a). We have only discussed large-scale features shown by the model. We emphasize that nevertheless our model suggests complex, rather small-scale and rapid sources of jerks, in accordance with the rapid fluctuations proposed for the fluid core by Olsen and Mandea (2008). Moreover, the SA animations show rather drastic changes starting with 1999 , with a very complicated pattern, when large acceleration and deceleration values co-exist.

Although this is near the end of the studied time interval and spline edge effects might occur, we note a coincidence with the very fast change observed in the north magnetic pole position, which is moving northwest toward Siberia by about $50 \mathrm{~km}$ each year (Olsen and Mandea, 2007b). Further studies of geomagnetic field behavior in all regions of the Earth with highest possible spatial and temporal resolution will be necessary to understand this complex behavior of the geodynamo. For most regions of the Earth, however, this was only been possible for less than a decade, as it can only be achieved from the combination of the observatory timeseries with data from the recent and upcoming satellite missions.

\section{Acknowledgements}

We thank Frank Lowes for helpful comments on an earlier version of the manuscript and to the suggestions of two anonymous refer- ees, which helped to improve the manuscript. Part of this study was performed during G.V. research visit at GFZ Potsdam, in the frame of the bilateral German-Croatian project: «Modelling the European geomagnetic secular variation on regional scale» sponsored by DAAD and the Croatian Ministry of Science, Education and Sport.

\section{REFERENCES}

Alexandrescu, M., D. Gibert, G. Hulot, J.-L. LeMouËL and G. SARACCo (1995): Detection of geomagnetic jerks using wavelet analysis, J. Geophys. Res., 100 (12), 557-12,572.

Chambodut, A., I. Panet, M. Mandea, M. Diament, M. HOLSCHNEIDER and O. JAMET (2005): Wavelet frames an alternative to spherical harmonic representation of potential fields, J. Geophys. Int., 163 (3), 875-899.

Courtillot, V., J. DucruiX and J.-L.LeMouë (1978): Sur une accélération récente de la variation séculaire du champ magnétique terrestre, (C.R. Acad. Sci. Paris. Ser. D 287), 1095-1098.

Courtillot, V. and J.-L.LeMouË (1984): Geomagnetic secular variation impulses, Nature, 311, 709-716.

DE BooR, C. (1978): A Practical Guide to Splines, (Springer-Verlag, New York).

De Santis, A., C. Falcone and J.M. Torta (1997): SHA vs. SCHA for modelling secular variation in a small region such as Italy, J. Geomag. Geoelectr., 49, 359-371.

Garcia, A., J.M. Torta, J.J. Curto and E. SAnClement (1991): Geomagnetic secular variation over spain 1970-1988 by means of sphericalcap harmonic analysis, Phys. Earth Planet. Inter., 68, 65-75.

HAINES, G.V. (1985): Spherical cap harmonic analysis, $J$. Geophys. Res., 90, 2583-2591.

HaINES, G.V. and L.R. NewITT (1986): Canadian geomagnetic reference field 1985, J. Geomag. Geoelectr., 38, 895-921.

Holschneider, M., A. Chambodut and M. Mandea (2003): From global to regional analysis of the magnetic field on the sphere using wavelet frames, Phys. Earth Planet. Inter., 135, 107-124.

KorTe, M. and R. Holme (2003): Regularization of spherical cap harmonics, Geophys. J. Int. 153, 253-262.

KotZÉ, P.B. (2001): Spherical cap modelling of Oersted magnetic field vectors over Southern Africa, Earth, Planets and Space, 53, 357-361.

LANGEL, R. (1987): The main field, in Geomagnetism, Vol 1, edited by J.A. JACOBS (Academic Press, Orlando), pp. 249-512.

Lesur, V., I. WARdinski, M. Rother and M. MANDEA (2008): GRIMM-The GFZ Reference Internal Magnetic Model based on vector satellite and observatory data, Geophys. J. Int., 173 (2), 382-394

Macmillan, S. (1996): A geomagnetic jerk for the early 1990's, Earth Planet. Sci. Lett., 137 (1), 189-192.

Macmillan, S., S. Maus, T. Bondar, A. Chambodut, V. Golovkov, R. Holme, B. Langlais, V. Lesur, F. 
Lowes, H. LÜHr, W. MaI, M. Mandea, N. Olsen, M. Rother, T. SABAKa, A. ThOMSON and I. Wardinski (2003): The 9th-generation International Geomagnetic Reference Field, Geophys. J. Int., 155, 1051-1056.

MAndeA, M., E. Bellanger and J.-L.LeMouË. (2000): A geomagnetic jerk for the end of the 20th century?, Earth Planet. Sci. Let., 183, 369-373.

Olsen, N., H. LÜhr, T. SabaKa, M. Mandea, M. Rother, L. TOFFNER-CLAUSEN and S. CHOI (2006): CHAOS-A model of the Earth's magnetic field derived from CHAMP, orsted, and SAC-C magnetic satellite data, Geophys. J. Int., 166 (1), 67-75.

Olsen, N. and M. MANDEA (2007a): Investigation of a secular variation impulse using satellite data: the 2003 geomagnetic jerk, Earth Planet. Sci. Lett., 255, 94-105.

Olsen, N. and M. MANDEA (2007b): Will the magnetic north pole wind up in Siberia?, American Geophysical Union, Fall Meeting 2007.

OlsEn, N. and M. MANDEA (2008): Rapidly changing flows in the earth's core, Nature Geosciences, 1, 390-394.

Olwer, F.W.J. and J.M. Sмith (1983): Associated Legendre functions on the cup, J. Computat. Phys., 51, 502-518.

SabaKa, T., N. Olsen and M. Purucker (2004): Extending comprehensive models of the Earth's magnetic field with Oersted and CHAMP data, Geophys. J. Int., 159, 521-547.

ThéBAULt, E. (2008): A proposal for regional modelling at the earth's surface, r-scha2d, Geophys. J. Int., 174 (1), 118-134.

ThéBault, E., J. Schott and M. Mandea (2006): Revised spherical cap harmonic analysis (r-scha): Validation and properties, J. Geophys. Res., 111, 1102.

Torta, J.M., A. García, J.J. Curto and A.D. Santis (1992): New representation of geomagnetic secular variation over restricted regions by means of spherical cap harmonic analysis: application to the case of Spain, Phys. Earth Planet. Inter., 74, 209-217.

VERBANAC, G. (2007): On regional modelling of the main geomagnetic field, Geofizika, 24, 1-27.

Verbanac, G., M. Korte and M. MAndea (2007a): On long-term trends of the European geomagnetic observatory biases, Earth, Planets and Space, 59 (7), 685 695.

Verbanac, G., H. LÜHr, M. Rother, M. Korte and M. MANDEA (2007b): Contribution of the external field to the observatory annual means and a proposal for their corrections, Earth, Planets and Space, 59 (4), 251-257.

WARDINSKI, I. and R. Holme (2006): A time-dependent model of the Earth's magnetic field and its secular variation for the period 1980-2000, J. Geophys. Res., 111, B12101, doi:10.1029/2006JB004401.

(received, February 4, 2009; accepted, July 9, 2009) 